\title{
Transformaciones y nuevas figuras de lo nervioso en la construcción de los relatos de Edgar Allan Poe
}

\author{
Transformations and new expressions of nervousness in the \\ construction of Edgar Allan Poe's short stories
}

\section{Francisco Pizarro Obaid}

Universidad Diego Portales. Santiago, Chile

francisco.pizarro@udp.cl

\section{RESUMEN}

El problema de la nerviosidad intervendrá de modo sistemático en la construcción de variados relatos de Edgar Allan Poe y pondrá en evidencia la influencia que ejerció en su obra la progresiva transformación que experimentó este concepto en el campo psicopatológico europeo y norteamericano durante el siglo XIX. El análisis de estas referencias llevará a cuestionar, por una parte, la existencia de una fuente inspiradora trascendente que subyacería al acto creador y, por la otra, las tradicionales interpretaciones biográficas que definían a sus rasgos patológicos de personalidad o a la fatalidad de su vida como las claves paradigmáticas para comprender la función de la angustia, la locura y la muerte en sus cuentos.

Palabras clave: Poe, cuento, nerviosidad, locura.

\section{ABSTRACT}

The problem of nervousness has a significant role to play in the construction of several of Edgar Allan Poe's short stories and shows the influence on his work of the gradual transformation of this concept in the field of psychopathology in Europe and North America during the nineteenth century. An analysis of these references raises questions not only about the existence of a transcendental source of inspiration 
underlying the creative act, but also about the traditional biographical interpretations that have defined his pathological personality traits, or the fatality of his life, as paradigmatic keys to understanding the function of anguish, madness, and death in his short stories.

Keywords: Poe, short story, nervousness, madness.

Recibido: 11.03.2011. Aceptado: 03.08.2011.

\section{INTRODUCCIÓN}

- ntre las diversas estrategias interpretativas que han guiado el análisis de Ela obra de Edgar Allan Poe, el expediente biográfico se convirtió en uno de los recursos más tradicionales en la comprensión de su legado literario. Bajo su hegemonía los aspectos más controvertidos de su historia personal -las complejas relaciones familiares y amorosas, los excesos referidos al opio, el alcohol y el juego-devinieron los referentes mayores en la tarea de explicar su particular predilección por el horror, la angustia y la muerte. Asimismo, los términos patología y genialidad no tardaron en transformarse en las categorías más recurrentes para devaluar la relevancia de sus escritos (Griswold, 1849) o, por el contrario, en las vías privilegiadas para solventar su originalidad (Dillon, 1911; Wood, J. 1926). Mediante el anudamiento de su vida y de su obra, Poe sería inmortalizado como una enigmática figura marcada por excepcionales y oscuras fuentes de inspiración ${ }^{1}$.

El recurso biográfico podría, sin lugar a dudas, constituirse como un material significativo en el análisis de una obra. Sin embargo, como advertirá Durand (2004), optar metodológicamente por un punto de inicio "en modo alguno implica, de hecho, que ese comienzo metodológico y lógico esté ontológicamente primero (...) Y si deliberadamente escogemos un punto de partida metodológico 'psicologista', de ninguna manera es para inmolarnos en un psicologismo ontológico" (48). Por lo tanto, la investigación de las motivaciones simbólicas que sustentan una obra "puede partir

${ }^{1}$ En concordancia con los supuestos freudianos que postulaban la necesaria participación de procesos psíquicos inconscientes en el origen de toda creación artística, Marie Bonaparte (1933) emprendió el monumental desafío de analizar la obra de Poe mediante los conceptos mayores del Psicoanálisis (inconsciente, represión, complejo de Edipo, etc.). Su trabajo dio lugar a una interpretación de Poe que, según Freud, arrojó luces para comprender "cuántos de los caracteres de su obra están condicionados por la peculiaridad del hombre, pero se averigua también que esta última es la sedimentación de intensas ligazones afectivas y vivencias dolorosas de su primera juventud" (229). 
indistintamente de la cultura o del natural psicológico, ya que lo esencial de la representación y del símbolo está contenido entre esos dos límites reversibles" (Durand, 2004: 45). En este sentido, como afirmará Starobinski (2008), una obra es una concordia discors pues su aprehensión sólo es posible al vincular la obra con "su origen psíquico, sus efectos remotos, el medio que la rodea. En esas circunstancias, lo indicios principales no vendrán del afuera, será en las obras mismas en sus repliegues donde se los encontrará, a condición de saber descubrirlos y leerlos" (34).

A partir de estas consideraciones resulta factible proponer el análisis de algunos cuentos de Poe para sostener que la dimensión psíquica involucrada en la construcción y la composición de sus relatos registrará, por una parte, las marcas de la crucial transformación que experimentarán los conceptos de nerviosidad y locura a lo largo del siglo XIX y, por la otra, evocará nociones vinculadas a la creación de instituciones hospitalarias públicas para el tratamiento específico de la enfermedad mental que tendrán lugar en aquel periodo.

De este modo, como afirmará Kristeva (1981), "todo texto se construye como mosaico de citas, todo texto es absorción y transformación de otro texto" (190), pero, como subrayará Barthes (1993), la meta "no es encontrar el sentido, ni siquiera un sentido del texto (...) nuestro objetivo es llegar a imaginar, a vivir lo plural del texto, la apertura de su significancia” (324). Así, cabría afirmar que si bien la vida de Poe estuvo marcada por la enfermedad y la fatalidad, psicopatología y nerviosidad, serán, a su vez, recursos teóricos y conceptuales que intervendrán -explícita o implícitamente- en la elaboración de sus relatos (short stories).

\section{LA HISTORIA DE POE Y LA COMPOSICIÓN DEL RELATO}

Dos días después de la muerte de Poe, Rufus Griswold (1849) -su albaceapublicará un obituario en el New York Tribune bajo el seudónimo de Ludwig. El documento constituyó la base para una posterior memoir que se transformaría en el texto de referencia para analizar la obra del poeta. Al suponer "una armonía singular entre sus cualidades personales y literarias" (Griswold, 1865: XLVII), la versión "oficial” sumergiría su multifacética producción literaria en el descrédito por un largo periodo, al exacerbar la función de los aspectos más polémicos de su biografía y su personalidad. No será sino hasta los años cuarenta que a través de la obra biográfica de Quinn (1941) se ofrezca una visión equilibrada entre la vida y la obra de Poe (Sova, 2007). 
Más allá de las diversas lecturas biográficas que contribuyeron a la creación de un mito, fue el propio Poe quien estableció en los últimos años de su vida algunas puntualizaciones tendientes a dilucidar la función que habrían tenido en sus escritos ciertos hechos personales catalogados de controvertidos. Ante las especulaciones de sus contemporáneos sobre cuáles serían los principios que definirían el fundamento de su obra refutará de manera enfática las críticas que ligaban su producción literaria con la tradición alemana consagrada al horror, al concluir que:

Si en muchas de mis producciones el terror ha sido la tesis, mantengo que ese terror no es de Alemania, sino del alma, -ya que he deducido ese terror únicamente de sus legítimas fuentes, y lo he impulsado solamente a sus resultados legítimos (Poe, 1840: 6).

En este sentido, Poe reconocerá, pese a todo, la participación de la dimensión vital en el proceso creativo e, incluso, hacia el final de su vida, ofrecerá una fórmula de sí mismo donde buscará describir sus rasgos más esenciales y su tormentosa relación con el alcohol:

Pero yo soy constitucionalmente sensible - nervioso en un grado muy inusual. Me volví loco, con largos intervalos de horrible sanidad. Durante estos accesos de absoluta inconsciencia, bebí, sólo Dios sabe cuán a menudo y cuánto. Habitualmente, mis enemigos refirieron mi locura a la bebida, más que la bebida a la locura (en Harrison, 1903: 287-288).

Indudablemente, confesiones como éstas podrían rendir crédito a las innumerables reseñas biográficas que hicieron de la dimensión patológica de su personalidad la llave maestra para comprender sus continuas referencias a figuras como el entierro prematuro, la fantasmagoría, las mutilaciones o las perversidades. Pese a ello, y en contraste con la aproximación biográfica, sus alusiones a la locura y lo nervioso intervendrán en la estructura misma de su obra literaria, ya no como una prolongación de su vida, sino como un instrumento conceptual destinado a potenciar el efecto ominoso de sus relatos. Como advertirá Ackroyd (2009) "fue el más calculador de los autores, por lo que nunca debería confundírsele con los narradores perturbados, e incluso psicóticos, de sus relatos. El Poe escritor buscaba con gran esmero los efectos más extremos" (83).

En su conceptualización de las condiciones que sustentan el acto crea- 
tivo, Poe intentará desenmascarar la figura popular y romántica del poeta al impugnar toda alusión a una fuente excepcional de inspiración y al proponer que el acto creador encontraría su fundamento en una operación de orden intelectual, sistemática y metódica. Denunciará que la épica moderna es un remedo de la antigüedad y declarará con vehemencia que "el tiempo de estas anomalías artísticas ha terminado" (Poe, 1850: 231). Bajo su perspectiva, toda creación tendría como meta la producción de un efecto en el lector y, en relación a ello, el principio fundamental de toda composición sería la brevedad. Una obra de arte debería ser juzgada, entonces, "más por la impresión que hace, por el efecto que produce, que por el tiempo que toma en imprimir el efecto o la cantidad de 'esfuerzo sostenido' que ha parecido necesario para efectuar la impresión” (Poe, 1850: 231-232).

La producción de un efecto devendrá una aspiración y una condición fundamental en la elaboración de toda obra, por lo que al analizar el proceso creativo con miras a enunciar sus mecanismos, puntualizará:

Me digo a mi mismo, en primer lugar, "De los innumerables efectos o impresiones, de los cuales son susceptibles el corazón, el intelecto, o (de manera más general) el alma, ¿cuál de ellos seleccionaré en esta ocasión? Habiendo elegido una novedad, primero, y en segundo lugar un efecto vívido, considero si es mejor obtenerlo mediante un incidente o el tono -ya sea por incidentes ordinarios y un tono peculiar, o a la inversa, o por la peculiaridad tanto del incidente y del tono- después de mirar a mi alrededor (o más bien dentro de mí) para tales combinaciones de evento o tono, que mejor me ayuden en la construcción del efecto" (Poe, 1846: 163).

Al considerar una dimensión histórico-conceptual destinada a explorar las posibles relaciones entre los términos empleados por Poe en sus relatos y otros campos del saber, quedará en evidencia cómo su obra será tributaria de las progresivas redefiniciones de lo nervioso y las concepciones de locura que registrará el campo médico europeo y norteamericano durante el siglo XIX. De este modo, la creación de tramas y personajes, cuyos rasgos fundamentales refieren a una particular alteración de la sensibilidad, harán de la nerviosidad y la locura un asunto de incidentes y tonalidades ${ }^{2}$.

\footnotetext{
${ }^{2}$ Baudelaire (1989) definirá a Poe como "el escritor de los nervios" (76), ya que con notable delicadeza pudo analizar de modo "minucioso y científico (...) todo lo imaginario que flota en torno al hombre nervioso y le conduce al mal” (77).
} 


\section{LA TRANSFORMACIÓN DE LO NERVIOSO Y SU INFLUENCIA EN EL CAMPO PSICOPATOLÓGICO}

Los discursos sobre el psiquismo desarrollados por el mesmerismo (Mesmer, 1779), la craneología (Gall, 1822) o la frenología (Spurzheim, 1818) tendrán una circulación significativa en los albores de la Psiquiatría moderna y constituirán una fuente significativa de inspiración para variados relatos de Poe (2009a, b, c, d, e, f), donde se evidenciará la presencia de aquellos principios teóricos (Hungerford, 1930; Schnackertz, 1999). Sin embargo, las nuevas consideraciones sobre la nerviosidad y la alienación desarrolladas a lo largo del siglo XIX atravesarán dichas referencias y servirán de fundamento para la renovación del conjunto del campo psicopatológico. Su influencia será constatable, asimismo, en los relatos de Poe.

Al describir la magnitud de las vicisitudes que registrará el concepto de lo nervioso, Gay (1999) afirmará que hasta 1800 -aunque vigente incluso hasta fines del siglo XIX o más- “"nervioso' o 'nerveux' significó vigoroso, contundente, fuerte y libre de todo tipo de debilidad; se habló, en alabanza, de la línea nerviosa de un hombre o de un caballo de carreras nervioso" (332). La dualidad en sus significados alcanzará también al ámbito médico hasta que finalmente se imponga su acepción moderna relativa a la enfermedad.

Desde una perspectiva médica, Shorter (1993) indica que la irritación espinal fue, hacia 1820, el principal recurso explicativo para los fenómenos de orden somático. Sus principios establecían que la irritación espinal local, a través de la acción refleja, determinaba la producción de signos patológicos y promovieron lo que más tarde sería conocido como Teoría refleja. Este modelo suponía que eran las redes nerviosas que recorren la columna vertebral las que "regulaban todos los órganos corporales, incluyendo el cerebro, con absoluta independencia de la voluntad humana. Si un órgano podía ejercer su influencia en sitios muy distantes, entonces las enfermedades en estos lugares podrían ser tratadas, interviniendo ese órgano" (Shorter, 1993: 40). Entre 1850 y 1900 la Teoría refleja "se convirtió en uno de los modelos médicos dominantes de la enfermedad nerviosa" (Shorter, 1993: 44).

Quizás la expresión más popular de estos supuestos se impuso en Norteamérica en la segunda mitad del siglo XIX a través de la categoría clínica de neurastenia (Beard, 1869), término que literalmente significa "falta de fuerza nerviosa”. La nueva entidad evolucionará de manera sostenida y se consolidará, incluso, bajo una fórmula identitaria, mediante el término ner- 
viosidad americana (Beard, 1881). La fatiga y el cansancio se erigirían, así, como los paradigmas de la nerviosidad y como las consecuencias directas de la acción patógena de la civilización moderna ${ }^{3}$.

Dos grandes innovaciones europeas relativas al estudio y el tratamiento de la alienación mental se verán influidas, a su vez, por los supuestos de la teoría refleja y se desarrollarán, principalmente, en el curso del siglo XIX. Por una parte, la locura será redefinida como un problema de orden médico, en tanto involucraría el compromiso de estructuras nerviosas y, por la otra, se asistirá a la progresiva creación de instituciones públicas destinadas al tratamiento específico de la alienación.

El impulso renovador, plasmado en los trabajos de William Battie (1758), Philippe Pinel (1801) y Jean Esquirol (1838), servirá de referencia a los desarrollos teóricos y terapéuticos que tendrán lugar en Norteamérica. El problema de los nervios sería abordado bajo los principios de la nueva racionalidad científica, giro que marcará su alejamiento de las concepciones hipocráticas y galénicas.

Battie (1758) refutará la concepción de un sistema nervioso conformado por una red de nervios huecos en los que transitarían elementos pneumaticos o espiritus animalis, para proponer que la causa de la percepción de objetos que no existen o que no se corresponden con los sentidos "debe ser algún desorden de la sustancia que es medular y estrictamente nerviosa" (41). La locura correspondería a delirios de orden sensorial y su característica más significativa referiría a la imaginación engañada, la cual conduciría a la percepción de objetos inexistentes o a la distorsión de sus cualidades. Su irrupción estaría promovida por una sobreexcitación nerviosa, seguida de una insensibilidad nerviosa, proceso bajo el cual se presentarían alucinaciones (Berrios, 2008). A partir de ello, serán distinguidos dos tipos de locuras, dependiendo de su causa: original y consecuencial. La primera refiere a un "trastorno interno de la sustancia nerviosa; la segunda se debe también a la misma sustancia nerviosa, siendo, de hecho, trastornada de modo semejante, pero alterada $a b$ extra; y, por lo tanto, debe ser principalmente atribuida a alguna causa remota y accidental" (Battie, 1758: 44).

En concordancia con la etiología orgánica promovida por Battie, Benja-

${ }^{3}$ Para Beard (1881) la neurastenia debía ser definida como un fenómeno inédito en la historia de la humanidad. Sostendrá, de manera enfática, que "todo esto es moderno y originalmente americano; en ninguna época, ni país, ni forma de civilización, ni Grecia, ni Roma, ni España, ni Holanda, en sus días de gloria, poseyeron tales enfermedades" (VII-VIII), ya que en dichos periodos estaban ausentes "la fuerza del vapor, la prensa periódica, el telégrafo, las ciencias y la actividad mental de la mujer" (VI), cambios que considera propios a lo que denominará civilización moderna, agente etiológico definitivo de la nueva entidad clínica. 
min Rush (1812) -consignado como el padre de la psiquiatría norteamericana- sostendrá que "la causa de la locura se asienta primariamente en los vasos sanguíneos del cerebro y, por eso, depende del mismo tipo de acciones mórbidas e irregulares que constituyen a otras enfermedades arteriales" (17). De este modo, la locura tendría su causa en el cuerpo y debería ser conceptualizada bajo los recursos de la ciencia biológica, lo que promovería el análisis de los fenómenos mórbidos bajo la óptica del organicismo.

La redefinición del problema de los nervios tendrá incidencia en su terapéutica. Durante la segunda mitad del siglo XVIII la incorporación y el tratamiento de enfermos mentales en el marco de los hospitales públicos de Norteamérica transformó a la locura en un asunto de orden médico y en un problema de interés gubernamental. En 1773 se consolidó en Williamsburg, Virginia, un asilo destinado específicamente al tratamiento de los alienados (Rosenkrantz, 2000).

El nuevo procedimiento de intervención terapéutica ideado por Pinel (1801) al alero de la inspiración revolucionaria, influirá progresivamente en el ámbito norteamericano. La tradición francesa lo había llamado "tratamiento moral", método consistente en "el arte de subyugar y domesticar, por así decirlo, al alienado, al ponerlo en estrecha dependencia de un hombre, que por sus cualidades físicas y morales, sea apropiado para ejercer sobre él un influjo irresistible y cambiar la cadena viciosa de sus ideas" (58). Su objetivo era un intento de humanizar y dignificar el tratamiento de la locu$r a$, tras siglos de oscuros procedimientos en los cuales estuvo ausente toda consideración médica del problema.

Los principio emanados de la tradición europea tuvieron eco en los escritos de Rush (1809), dando lugar, en el marco de la discusión médica, a la proposición del concepto de facultad moral, la "capacidad en la mente humana de distinguir y elegir lo bueno y lo malo, o, en otras palabras, virtud y vicio" (172). En oposición a la tradición metafísica, el problema moral tendría ahora su fundamento en la acción y la volición, giro que impondría la necesidad de conocer "la influencia de causas físicas sobre el poder moral de la mente" (174).

El asilo se transformaría en el espacio para intervenir sobre las variables que han desequilibrado las facultades humanas mediante la correcta administración del ambiente, la conducta y el cuerpo. Desde un punto de vista sociocultural "lo que era psiquiatría biológica para los médicos, era nervios para los pacientes. A comienzos del siglo diecinueve, las familias traían a los pacientes al asilo, no por locura, sino por la imputación de enfermedad nerviosa" (Shorter, 1997: 113). 
El alcance que tendrán las transformaciones teóricas y las innovaciones clínicas asociadas a lo nervioso cruzarán las fronteras de la medicina para inscribirse en diversas manifestaciones socioculturales. La creación literaria no será la excepción e incorporará sus referencias como quedará de manifiesto en los escritos de Poe.

\section{LA NERVIOSIDAD Y LA LOCURA EN LA CONSTRUCCIÓN DEL EFECTO INQUIETANTE}

La intervención literaria de los nuevos supuestos acerca de la nerviosidad será administrada de manera estratégica por Poe en algunos de sus relatos, donde narradores y personajes estarán marcados por una estructuración psíquica singular.

Quizás la figura más expresiva de esta particular condición sea la del narrador que con inusual pulcritud ha matado y enterrado al viejo del "ojo de buitre", invitando al lector a seguir con atención la crónica de su crimen y su inesperada confesión. Su interpelación será vehemente desde las primeras líneas del relato: "Es cierto! Siempre he sido nervioso, muy nervioso, terriblemente nervioso ¿Pero por qué afirman ustedes que estoy loco? La enfermedad había agudizado mis sentidos, en vez de destruirlos o embotarlos" (Poe, 2009g: 135).

El problema de lo nervioso retornará en un nuevo relato. Se trata del enigma del brigadier John A. B. C. Smith, aparente prototipo de la belleza masculina, quien literalmente será "el hombre que se gastó". La progresiva transformación de su imagen producirá un efecto ominoso en su admirador, quien se verá súbitamente confrontado al develamiento del secreto que sostenía ese cuerpo que ofrecía perfección. Al iniciar la historia, el narrador advertirá: "Soy constitucionalmente nervioso-; es un defecto de familia, y no lo puedo impedir. La menor apariencia de misterio, la cosa más ínfima que no alcance a comprender, bastan para sumirme de inmediato en un estado de lamentable agitación" (Poe, 2009h: 715).

Bajo otra perspectiva, un nuevo cuento presentará al señor Valdemar como sujeto experimental de la hipnosis in articulo mortis. Los procedimientos implementados por los investigadores buscarán comprobar si un moribundo es susceptible de influencia magnética y si el arte hipnótico lograría detener la intrusión de la muerte. Además de haber obtenido las autorizaciones correspondientes por parte del enfermo, el hombre cumplía un requisito esencial: poseía un "temperamento muy nervioso, que le convertía 
en buen sujeto para experiencias hipnóticas" (Poe, 2009e: 120).

Poe (2009i) transportará un nuevo relato a la Antigüedad con el fin de interiorizar al lector sobre lo ocurrido en la extraña cita celebrada en la sombría ciudad de Ptolemáis, donde un grupo de nobles sobrevivientes de la peste que había asolado el imperio, se sumergen en un espacio lujurioso y mortífero; una atmósfera inquietante que actúa sobre el colectivo llevará al narrador a describir su condición como un "terrible estado de la existencia que alcanzan los seres nerviosos cuando los sentidos están agudamente vivos y despiertos, mientras las facultades yacen amodorradas" (272).

Pero no serán sólo las modernas nosografías o los nuevos supuestos que rigen las concepciones etiológicas de la enfermedad nerviosa de su época los que aportarán a la construcción del relato. Poe evidenciará también un significativo grado de actualización respecto de las emergentes teorizaciones del discurso psicopatológico sobre la alienación y sus formas de tratamiento, lo que quedará plasmado en un magnífico cuento referido directamente al tratamiento de la locura.

El narrador detallará las extrañas experiencias que tuvieron lugar en su visita a una Maison de Santé o manicomio privado en Francia. En el encuentro con los internos, y en su aproximación al conocimiento de los procedimientos curativos en boga, tendrá ocasión de instruirse sobre las diferencias entre el sistema de la dulzura y el sistema del doctor Tarr y del profesor Fether, método alternativo a la tradicional intervención vinculada al factor moral, usualmente empleada en la Institución. Había sabido que en el sistema de la dulzura "los castigos estaban abolidos, que se prescindía en casi todos los casos del confinamiento, y que los pacientes, aunque secretamente vigilados, gozaban de gran libertad aparente, permitiéndoseles que pasearan por la casa y los jardines con todos los derechos de las personas en su sano juicio" (Poe, 2009j: 800). Monsieur Maillard, quien será presentado como director del establecimiento, informará al visitante que las ventajas del sistema de la dulzura habían sido sobredimensionadas. Finalmente, representaba grandes peligros, razón por la cual había sido necesario volver a los antiguos métodos.

El sistema de la dulzura develará su particularidad en la progresiva transformación del relato. Maillard, tras enloquecer, se había convertido en paciente y había previsto un novedoso sistema, al que calificaba de "verdaderamente extraordinario... muy sencillo... pulcro... nada complicado... realmente delicioso..." (800). En complicidad con sus nuevos colegas había emprendido una revolución contra los guardianes, quienes, dominados por los locos, habían sido primeramente untados de alquitrán, luego empluma- 
dos y finalmente metidos en las celdas subterráneas ${ }^{4}$.

El abordaje del factor moral no se agotará para Poe en su dimensión institucional. En un nuevo relato expondrá una crítica directa al mesmerismo y la frenología, para luego acuñar una expresión inédita tendiente a conceptualizar ese primum mobile que estaría en el fundamento de las más primordiales expresiones de lo humano: el demonio de la perversidad; sentimiento radical, primitivo e irreductible, ignorado y olvidado por las ciencias de la mente y los moralistas.

El narrador denunciará las omisiones de los frenólogos en la tarea de consignar las funciones de lo humano y los órganos asociados a ellos; se concentraron en el hambre, al identificar un órgano de la alimentividad y en el amor, con su consiguiente órgano de la amatividad; luego engrosaron la lista con la combatividad, la idealidad, la casualidad, la constructividad, es decir, con "todos los órganos que representaran una tendencia, un sentimiento moral o una facultad del puro intelecto" (Poe, 2009f: 191). En esta empresa, los spurzheimistas siguieron a la tradición e, incluso, a los preceptos divinos. Desconocieron y negaron aquel factor esencial denominado el demonio de la perversidad, un móvil, un impulso, a partir de cuyas incitaciones "actuamos sin objeto comprensible, o, si esto se considera una contradicción en los términos, podemos llegar a modificar la proposición y decir que bajo sus incitaciones actuamos por la razón de que no deberíamos actuar" (192). La incidencia de este inédito factor confrontará al lector con el acto criminal y el sorprendente desenlace animado por la culpa y el deseo de autopunición del narrador.

Pero el interés de Poe no sólo recaerá sobre las figuras más evidentes de la locura y la nerviosidad sino que, incluso, llegará a interrogar la condición humana, al emprender el análisis crítico de las relaciones entre progreso, civilización y sufrimiento. Una sugerente reflexión será expuesta con motivo del extraordinario coloquio celebrado por Monos y Una.

En una atmósfera nostálgica y sideral, el lector asistirá al diálogo de los amantes, quienes, reunidos siglos después de su muerte, teorizarán sobre el pasaje hacia la muerte y denunciarán los extravíos de la humanidad. En aquellos tiempos "Como el hombre no podía dejar de reconocer la majestad de la Naturaleza incurría en pueriles entusiasmos por su creciente dominio sobre los elementos de aquélla. Mientras se pavoneaba como un dios en su propia fantasía, lo dominaba una imbecilidad infantil” (Poe, 2009k: 365).

\footnotetext{
${ }^{4}$ Respecto de los nombres Dr. Tarr y Prof. Fether, que designan al sistema, el Traductor refiere: "De tar, alquitrán, $y$ feather, pluma. To tar and feather significa untar a alguien con alquitrán y cubrirlo luego de plumas". (Poe, 2009j: 799).
} 
El conocimiento habría permitido logros excepcionales, pero al mismo tiempo, en sus diversas aplicaciones utilitarias, habría conducido inevitablemente al sufrimiento, el malestar y la muerte.

De este modo, el problema de la nerviosidad no sólo invocará la dimensión horrorosa en los relatos de Poe, sino que igualmente será la vía para analizar la paradójica ilusión que promueve la idea del progreso, supuesto avance que no hace sino poner en evidencia un factor irreductible de la subjetividad humana que impide la armonía, la totalidad o la unidad. Al intentar nombrar ese determinismo que la ciencia, la religión y la filosofía intentaron conceptualizar sin resultados, Poe lo llamará el demonio de la perversidad.

\section{DISCUSIÓN}

Al distanciarse de las referencias exclusivamente biográficas y dar paso a la consideración de los problemas conceptuales involucrados en las referencias a lo nervioso y la locura en la obra de Poe, surge un entramado textual donde se entrecruzan los discursos de la medicina y la literatura. De este modo, si bien es posible constatar la presencia de elementos psicopatológicos en su personalidad o consignar una historia familiar y amorosa marcada por el conflicto y la fatalidad, sus escritos darán testimonio de un proceso productivo, metódico y sistemático, que incorporará las nuevas concepciones sobre la enfermedad nerviosa que iniciaron su redefinición a comienzos del siglo XIX.

La nerviosidad será tratada bajo los nuevos criterios de composición desarrollados por Poe. Buscará desacreditar toda alusión a fuentes de inspiración sublimes o divinas y refutará la tradicional versión idealizada del poeta, que encontraría su iluminación en una fuerza inefable. Se trata de un proceso sistemático y racional que busca, en una extensión breve, producir un efecto.

Las nuevas teorizaciones europeas acerca de la enfermedad mental servirán de fuente inspiradora para la psiquiatría norteamericana. Las concepciones de la nerviosidad imperantes en el curso del siglo XIX llevarán a situar los desvíos de la razón en una afección de los nervios, fundamento a partir del cual podrían ser explicados el mundo delirante y alucinatorio, así como una amplia gama de trastornos referidos a la sensibilidad y la percepción. En un registro terapéutico, las nuevas prácticas encontrarán inspiración en el tratamiento moral, método elaborado al alero de las ideas revolucionarias, que entre sus objetivos buscaba la dignificación del hombre y redefinir a la 
locura como una enfermedad que podría ser curada a través de intervenciones en el cuerpo, el espacio institucional y mediante la particular relación emprendida entre el alienado y una figura competente que pudiera inspirar respeto y admiración. Su objetivo era reconducir al enfermo al primado de la razón.

De este modo, lo nervioso resultará un recurso fértil para la producción de un efecto ominoso e inquietante, procedimiento que puede ser confirmado en algunos de sus relatos donde, en el marco del horror, la angustia y la muerte, sus personajes estarán marcados por la nerviosidad, condición que los llevará a franquear los límites de la razón para abandonarse a los crímenes, la culpa y la autopunición.

La exploración del factor moral no se agotará en el registro de un relato fantasmagórico, sino que encontrará un tratamiento novedoso bajo la forma de una reflexión teórica, un análisis científico o un debate entre discursos. Su tratamiento de la nerviosidad, alcanzará incluso una profunda reflexión sobre la cultura y el avenir, al poner entre paréntesis la idea del progreso que la modernidad pretendió erigir como clave de un futuro promisorio.

\section{REFERENCIAS}

Ackroyd, P. 2009. Poe: Una Vida Truncada. Barcelona: Edhasa.

Barthes, R. 1993. La aventura semiológica. Barcelona: Paidós.

Battie, W. 1758. A treatise on madness. London: J. Whiston, and B. White.

Baudelaire, Ch. 1989. Edgar Allan Poe. Madrid: Visor.

Beard, G. M. 1869. "Neurasthenia, or nervous exhaustion”, en The Boston Medical and Surgical Journal, pp. 217-221.

Beard, G. M. 1880. A practical treatise on nervous exhaustion (neurasthenia). New York: W. Wood \& Co.

Beard, G. M. 1881. American Nervousness Its Causes And Consequences. A Supplement to Nervous Exhaustion (Neurasthenia). New York: G. P. Putnam's Sons.

Berrios, G. 2008. Historia de los sintomas de los trastornos mentales. La psicopatología descriptiva desde el siglo XIX. México: Fondo de Cultura Económica. Bonaparte, M. 1933. Edgar Poe. Paris: Denoël et Steele.

Dillon, J. 1911. Edgar Allan Poe. His Genius and Character. New York: The Knickerbocker Press.

Durand, G. 2004. Las estructuras antropológicas del imaginario: Introducción a la arquetipología general. México: Fondo de Cultura Económica. 
Esquirol, J. 1838. Des maladies mentales considérées sous les rapports médical, hygiénique et médico-légal. Paris: J.-B. Baillière.

Freud, S. 1996. "Prólogo a Marie Bonaparte, Edgar Poe, étude psychanalytique (1933)". Trad. José L. Etcheverry. Ed. James Strachey. En Obras completas Sigmund Freud. v. XXII. Buenos Aires: Amorrortu.

Gall, F. J. 1822. Sur l'origine des qualités morales et des facultés intellectuelles de l'homme, et sur les conditions de leur manifestation. Paris: Béchet.

Gay, P. 1999. The bourgeois experience: Victoria to Freud. Vol. 2. New York: W.W. Norton \& Co.

Griswold, R. 1849. "Death of Edgar A. Poe", en New-York Daily Tribune, October 9, p. 2, cols. 3-4.

Griswold, R. 1865. Memoir, in The Works of Edgar Allan Poe, Vol. 1. New York: W. J. Widdleton.

Harrison, J. A. 1903. Life and letters of Edgar Allan Poe. Vol. 2. New York: T.Y. Crowell \& Co.

Hungerford, E. 1930. "Poe and Phrenology", en American Literature 2, 3, pp. 209-231.

Kristeva, J. 1981. Semiótica 1. 4a ed. Madrid: Editorial Fundamentos.

Mesmer, F. 1779. Mémoire sur la découverte du magnétisme animal. Paris: P. Fr. Didot.

Pinel, P. 1801. Traité médico-philosophique sur l'aliénation mentale ou la manie. Paris: Richard, Caille et Ravier.

Poe, E. A. 1840. Tales Of The Grotesque And Arabesque. Vol. I. Philadelphia: Lea And Blanchard.

Poe, E. A. 1846. "The philosophy of composition", en Graham's [American Monthly] Magazine 28, 4, January 01. In Edgar Allan Poe Society of Baltimore. [En línea] Disponible en: http://www.eapoe.org/works/essays/philcomp.htm. Consulta: 27.09.2011.

Poe, E. A. 1850. "The poetic principle", en Sartain's Union Magazine of Literature and Art, 7, 4m January 01. In Edgar Allan Poe Society of Baltimore. http://www.eapoe.org/works/essays/poetprnc.htm. [En línea] Disponible en: http://www.eapoe.org/works/essays/philcomp.htm. Consulta: 27.09.2011.

Poe, E. A. 2009a. "El hombre de negocios". En J. Cortázar (Trad.). Cuentos completos. Madrid: Páginas de Espuma, pp. 949-956.

Poe, E. A. 2009b. "Un cuento de las Montañas Escabrosas”. En J. Cortázar (Trad.). Cuentos completos. Madrid: Páginas de Espuma, pp. 179-187.

Poe, E. A. 2009c. "Revelación mesmérica”. En J. Cortázar (Trad.). Cuentos completos. Madrid: Páginas de Espuma, pp. 335-346. 
Poe, E. A. 2009d. "Conversación con una momia”. En J. Cortázar (Trad.). Cuentos completos. Madrid: Páginas de Espuma, pp. 597-611.

Poe, E. A. 2009e. "La verdad sobre el caso del Señor Valdemar". En J. Cortázar (Trad.). Cuentos completos. Madrid: Páginas de Espuma, pp. 119-127.

Poe. E. A. 2009f. "El demonio de la perversidad". En J. Cortázar (Trad.). Cuentos completos. Madrid: Páginas de Espuma, pp. 191-196.

Poe, E. A. 2009g. "El corazón delator". En J. Cortázar (Trad.). Cuentos completos. Madrid: Páginas de Espuma, pp. 137-141.

Poe. E. A. 2009h. "El hombre que se gastó". En J. Cortázar (Trad.). Cuentos completos. Madrid: Páginas de Espuma, pp. 715-723.

Poe, E. A. 2009i. “Sombra”. En J. Cortázar (Trad.). Cuentos completos. Madrid: Páginas de Espuma, pp. 271-273.

Poe, E. A. 2009j. "El sistema del doctor Tarr y del profesor Fether". En J. Cortázar (Trad.). Cuentos completos. Madrid: Páginas de Espuma, pp. 799-814. Poe, E. A. 2009k. "El Coloquio de Monos y Una”. En J. Cortázar (Trad.). Cuentos completos. Madrid: Páginas de Espuma, pp. 363-370.

Quinn, A. H. 1941. Edgar Allan Poe, a critical biography. New York: D. Appleton-Century Co.

Rosenkrantz, B. 2000. “Estados Unidos”. En J. Postel \& C. Quétel. Nueva historia de la psiquiatría. México: Fondo de Cultura Económica.

Rush, B. 1809. Medical inquiries and observations. (3a Ed.), Vol. 1. Philadelphia: Mathew Carey.

Rush, B. 1812. Medical inquiries and observations upon the diseases of the mind. Philadelphia: Kimber \& Richardson.

Schnackertz, H. J. 1999. "Of Bumps and Brains: E. A. Poe and Phrenology". En Lost worlds \& mad elephants: Literature, science and technology, 17001990. Glienicke, Berlin: Galda + Wilch.

Shorter, E. 1993. From paralysis to fatigue: a history of psychosomatic illness in the modern era. New York: Free Press.

Shorter, E. 1997. A history of psychiatry: From the era of the asylum to the age of Prozac. New York: John Wiley \& Sons.

Sova, D. 2007. Critical Companion to Edgar Allan Poe. A Literary Reference to his Life and Work. New York: Facts on File.

Spurzheim, G. 1818. Observations sur la phrénologie. Paris: Truettel \& Würtz. Starobinski, J. 2008. La relación crítica. Buenos Aires: Nueva Visión.

Wood, J. 1926. Edgar Allan Poe. A study in Genius. New York: Alfred A. Konpf. 